\title{
Automated Near-Field Scanning to Identify Resonances
}

\author{
Muchaidze, Giorgi ${ }^{(1)}$, Huang Wei ${ }^{(2)}, \operatorname{Jin}_{\mathrm{Min}^{(1)}}$, Shao Peng ${ }^{(2)}, \operatorname{Jim}_{\text {Drewniak }}{ }^{(2)}$ and David Pommerenke ${ }^{(2)}$ \\ (1) Amber Precision Instruments \\ Santa Clara, CA, USA, Email: jinmin@amberpi.com \\ ${ }^{(2)}$ Missouri Science and Technology (former University Missouri Rolla) \\ Rolla, MO, USA, Email: davidjp@mst.edu
}

\begin{abstract}
Near-field scanning systems are a tool for rootcause ESD, EMI, and immunity analysis of electronic systems, as well as qualification methodology for ICs and modules. For emissions, they have developed into a standardized method. Development of universally accepted file formats for data exchange is on-going. Four main types of scanning have been implemented by this and other authors: Near-field EMI scanning, ESD scanning, radiated immunity scanning, and resonance scanning. This article concentrates on resonance scanning as a newly added method for automated EMC system analysis.
\end{abstract}

Near field scanning; ESD; resonance; EMI

\section{INTRODUCTION}

EMC tests are performed on a system level, and thus do not reveal the root cause of a problem. Root-cause analysis is the daily bread of EMC engineers. Consequently, a wide variety of analysis methods have been developed, one of them is near field scanning. It finds application in emissions, immunity and coupling analysis:

- Near field electromagnetic interference (EMI) measurement [1]-[3]

- RF immunity scanning using modulated sine wave [4]

- $\quad$ ESD scanning [5]

- Resonance scanning.

The challenge in all of the scanning methods is relating local results to system level results. For example, if a clock frequency leads to strong emissions and near field scanning is performed, the clock traces will show up strongly. This indicates that the clock is the source, however it does not reveal that the areas of strong field at the clock frequency are causing the EMI problem (as they do not show the coupling path). Often, in EMI analysis, it is not strong fields that indicate a problem, but weak fields in areas where there should be none. Such an analysis requires system-level EMI knowledge, and cannot be directly implemented in software.
Immunity scanning shows areas where coupling to devices (either directly or via traces or cables) leads to disturbances. However, from the scan maps one cannot directly conclude that those areas are causing an observed system level failure. A highly noise sensitive integrated circuit (IC) in the middle of a board, being connected by short traces will probably not lead to a system upset, relative to a relatively robust IC that is connected to badly routed reset lines connected via unshielded flex cables. The examples above illustrate the difficulty in connecting local results to system results.

Many authors have devoted their research to closing this gap. For example, using near-field to far-field transformation to predict EMI from scan [6], using near field scan data to predict TEM cell measurement data that again can be connected to the far field [7], or using near field data as excitation in numerical models.

Near-field scanning shows locally strong fields, however, in most cases they do not identify the aspects of the printed circuit boards (PCB) or system design that lead to a failure. In this regard, near-field scanning is more of a source identification tool than a coupling path identification tool.

Identifying the coupling paths is the "holy grail" of EMC analysis. Resonant coupling paths are especially important, as they will couple energy very efficiently at the resonance frequency. Identification of resonances has been an important aspect of EMC analysis for decades. This article briefly reviews other scanning methods, and then presents an automated resonance scanning method.

\section{EMI SCANNING}

EMI scanning probes the local electric or magnetic field above an IC, PCB, or around a equipment under test (EUT). The data are usually used for identifying areas of strong magnetic or electric field. An implicit, but often incorrect, assumption is made that an area of strong field is the cause of EMI problems. The near-field information is used as follows:

- Identifying the cause of an EMI problem 
- Comparing two boards or after a modification to the original case

- Near-field - far-field transformations

- Identification of current paths

- Capturing data to be used as an excitation in a numerical simulation

\section{IMMUNITY/ESD SCANNING}

In immunity scanning the reaction of the EUT is tested. RF is injected into the EUT at different locations. Thus, for automatic scanning, a feedback needs to be established between the EUT and the scanning controller, such that the scanning controller "knows" if an error has occurred. The difference between immunity scanning and ESD scanning is the type of noise injected. Modulated RF signals are usually injected in immunity scanning, whereas narrow pulses are injected in ESD scanning. The injection usually takes places via the electric or the magnetic field, however direct injection techniques are possible.

\section{RESONANCE SCANNING}

The challenge in EMC analysis is often locating the coupling paths and, to a lesser degree, locating the antennas. The most basic coupling theory for EMI predicts a broadband, or linear with increasing frequency, coupling strength. These models do not intend to take the complexity of real systems into account. Their use lies in the illustration of basic principles, and their direct application is limited to simple cases on PCBs or cases with well controlled field structures as they can be found in the TEM cell tests (IEC61967).

\section{A. Are resonances important?}

The most basic coupling mechanisms let us expect smooth (e.g. $20 \mathrm{~dB} /$ decade) coupling behavior. In practice, however, this smooth frequency behavior is not observed. For example, experience in immunity testing (IEC61000-4-3) of systems has shown that immunity failures are usually not of broadband nature, but rather occur in a relatively narrow frequency range. ESD is a pulsed, broadband disturbance, however EUTs often only respond to a narrow portion of the spectrum [8]. If emission testing is performed while the clock frequencies are varied over a wide range, we observe strong peaking in the radiated signals. All these examples are indicative of internal system resonances. At resonance frequencies, the EMI radiation or immunity sensitivity may increase by orders of magnitude. This signifies the importance of locating system resonances.

\section{B. Where do resonances occur?}

Resonances can be of lumped (L-C) or distributed nature. From smallest scale to largest scale, resonances may be found at:
- IC power distribution resonances. The on-die capacitance and the interconnect inductance form an LC circuit, peaking the on-die power-ground noise at frequencies from about $10-1000 \mathrm{MHz}$

- PCB power distributions form resonances. At lower frequencies by the interaction of decoupling capacitances, ESL and distributed inductances, at higher frequencies by the propagation delay within power traces or planes.

- Traces on the PCB can resonate if not terminated at one end at least.

- Ground fills of PCB can resonate if they are long and narrow, cross slots and not connected with sufficient vias to a ground plane [9].

- PCBs that are connected via cables or flex circuits to other PCBs form resonators

- Cables resonate

- Heat-sinks

- Structural elements, especially if they are long and thin

- Enclosures

- Cables on enclosure, power cables, I/O lines

On one side these resonances can couple to local voltages and currents, on the other side they can form antennas and couple to the far field. Thus, they need to be regarded as the link between effects seen in near field EMI scanning and the far field. In case of immunity testing the resonances will be excited by the far field and cause strongly, resonance enhanced voltages and currents increasing the likelihood of a failure at the resonance frequencies.

\section{How to identify resonances}

Resonance scanning is performed with the EUT turned off. It is a two port measurement (e.g. performed using a network analyzer). Different configurations are known: Far-field to far-field coupling as seen in resonances in radar cross sections to the well known grid dipper as used by ham radio operators. Figs. 1-3 illustrate three methods suitable for automated scanning:

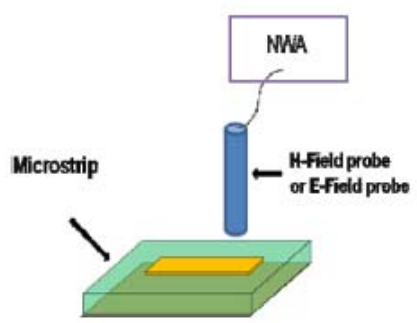

Figure 1 Identifying resonances via S11 measurements 


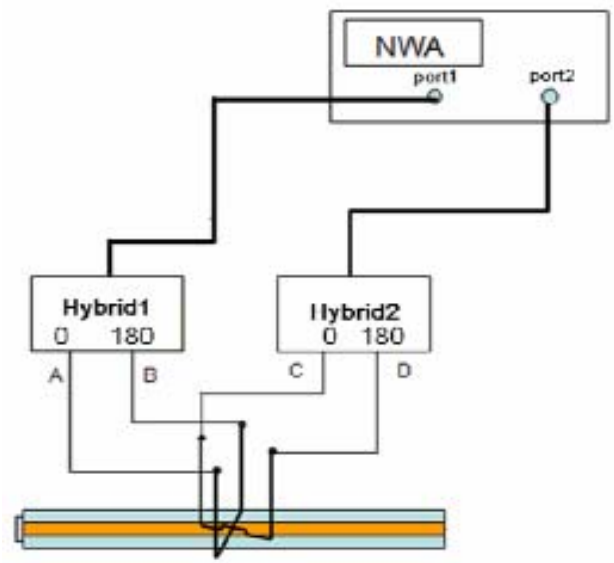

Figure 2 Identifying resonances via two orthogonal probes [10]

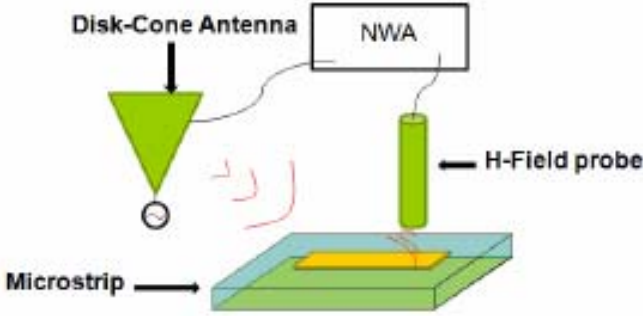

Figure 3 Identifying resonances via a far field probe and a local probe.

A variety of probing methods can be used. Fig. 1 depicts a measurement method based on S11. It is similar to the grid dipper used for decades by ham radio operators. If there is no resonating structure for the probe to couple to, most of the RF energy injected into the probe will be reflected by the probe. If the probe is able to couple to a resonating structure, however, less energy will be reflected leading to small dip in the S11 value. This method couples and decouples locally, so it is more suitable to find local resonances, no matter how well they coupled to the far field. Other methods use two probes that are decoupled to each other, e.g., by their field structure [10], distance or field component. The network analyzer measures S21 which expresses the coupling from one probe to the other. If resonating structures can couple to both probes an increased S21 value will be observed at the resonance frequency. The method moves both probes to different locations while scanning. The method couples and decouples locally, however, as both probes do not have to have the same size and distance, one can adjust the amount far field or near field coupling. A third method does not use a local excitation but illuminates the EUT from an antenna placed at some distance, thus, only one probe moves. The moving probe measures the local magnetic field, excited by the far field and resonances are again identified by an increased S21.

\section{Measurements on test structure}

A set of test structures have been created to investigate different probing and data analysis techniques. An example test structure, partially overlaid by a test result is shown in Fig. 4.

The test structure of Fig. 4 consists of four traces on a $1.6 \mathrm{~mm}$ thick PCB. The traces resonate at frequencies between $380 \mathrm{MHz}$ and $800 \mathrm{MHz}$. As other test structures thinner PCBs have been used increasing the difficulty coupling to the traces. To test if resonances in real systems can be identified an open iMac computer is used. The first level of data analysis plots the magnitude of the S21 response over the locations. Fig. 5 shows the result for the $1.6 \mathrm{~mm}$ PCB test structure.

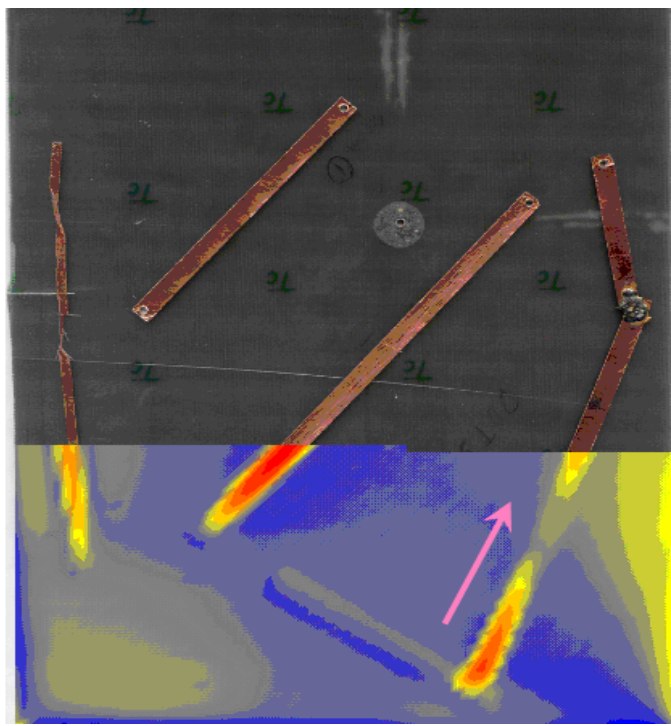

Figure 4 Test structure for resonance scanning partially overlayed with a scan result.

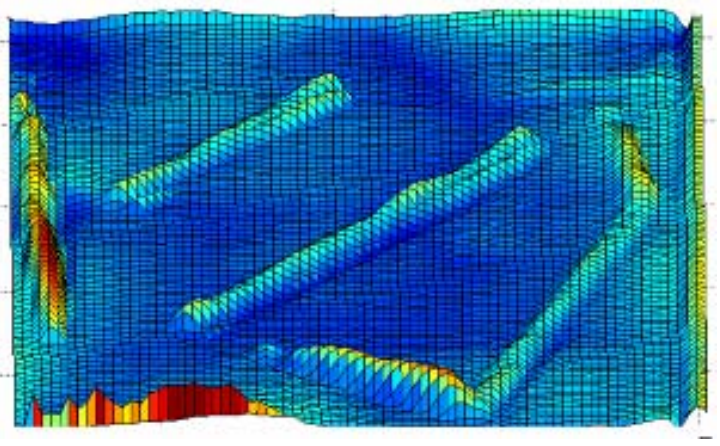

Figure 5 Test structure for resonance scanning partially overlayed with a scan result.

The next step in data analysis determines the frequency at which the resonance occurs, and associates the vertical axis with the frequency (see Fig. 6 ). The peaks are identified by subtracting a reference (probe at a location having no resonance) from the measured S21 parameters. 
The peaks are identified and classified according to their Q-factor, resonance frequency and harmonic relationship.

The methodology was applied to an iMac computer, shown in Fig. 7.

The cable indicated by the arrows experiences a resonance at around $100 \mathrm{MHz}$. An analysis, showing the measured probe-to-probe response as a function of frequency, is shown in Fig. 8. It clearly reveals the cable resonance around $100 \mathrm{MHz}$.

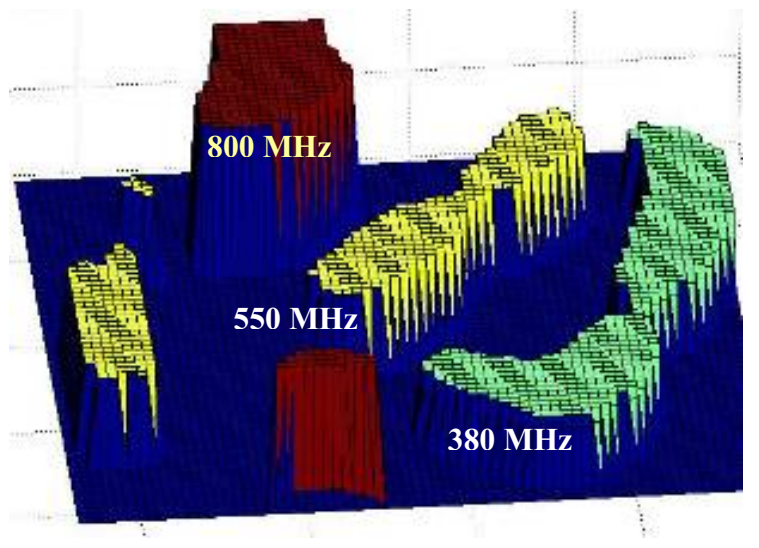

Figure 6 Itendified resonance frequencies for the test structure. Directions $\mathrm{X}$ and $\mathrm{Y}$ identify the location, the $\mathrm{Z}$-axis identifies frequency.

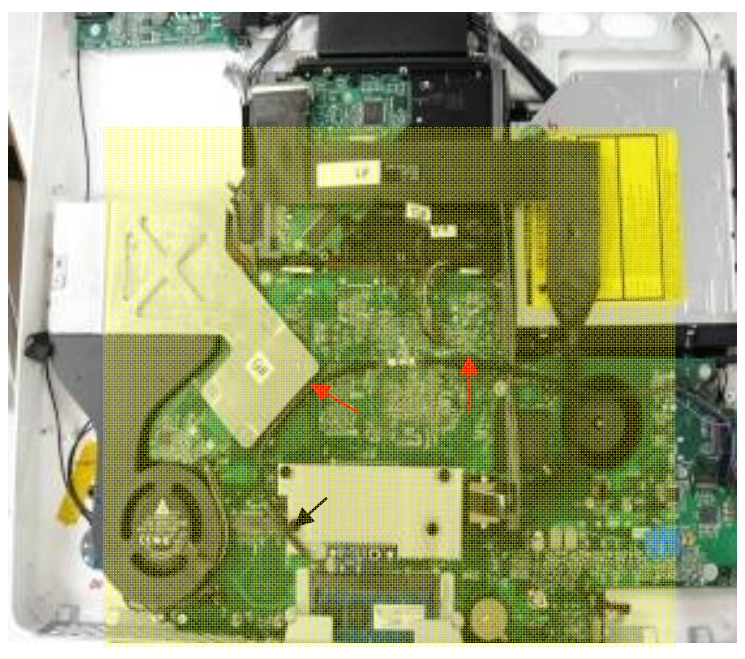

Figure 7 Resonance scanning test object iMac. The dotted box shows the scan area

\section{SUMMARY}

Scanning methods are a tool of growing importance for EMI and immunity analysis. Their main advantage is their ability to assist in root cause analysis. This paper has introduced resonance scanning as a method to identify system resonances. These resonances form the link between local effects and the far-field. The method extends known resonance identifying techniques to automatic scanning, taking into account the particularities of automated scanning.
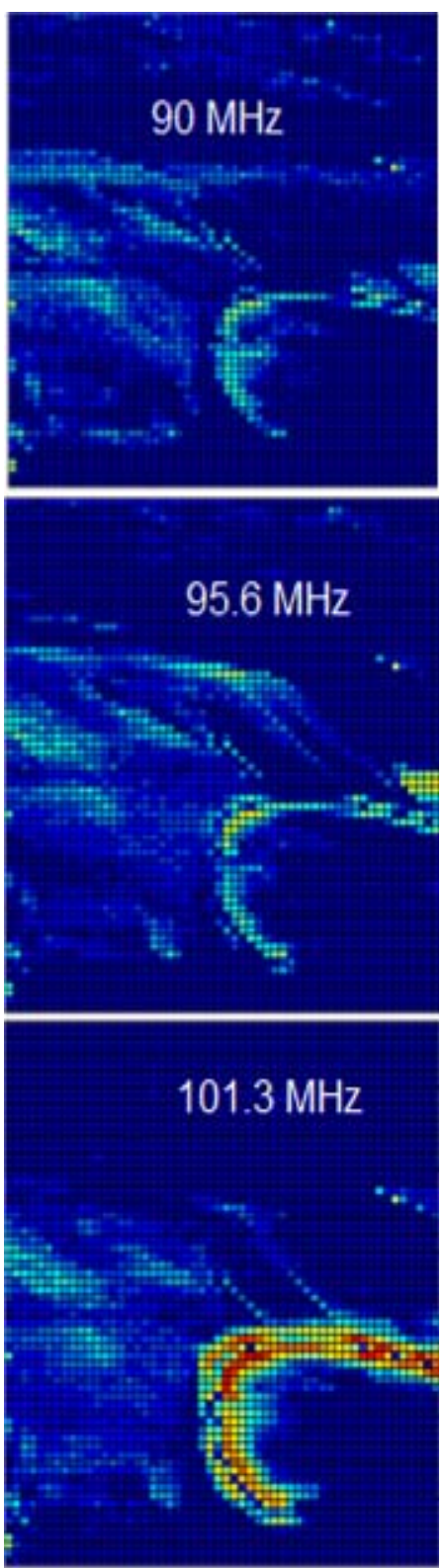

Figure 8 Scan magnitude results for the iMac at 90, 95.6 and 101.3 MHz showing the resonance of the cable. 


\section{REFERENCES}

[1] Haixiao Weng, Jin Shi, Daryl Beetner, and Richard E. DuBroff, "Compensation and calibration of near field scan measurements for EMC analysis, diagnosis and prediction," 3rd International Conference Proceednings Electromagnetic Near-Field Characterization \& Imaging, St. Louis, Missouri USA, June $27-$ 292007 .

[2] T. Harada, N.Masua, and M. Yamaguchi, "Near-field magnetic measurements and their application to EMC of digital equipment," IEICE Trans. Electron. Vol. E89-C, No. 1, pp. 9-15, Jan. 2006.

[3] A. Boyer, S. Bendhia, E, Sicard, "Characterisation of electromagnetic susceptibility of integrated circuits using nearfield scan," Electron. Lett., Overstress/Electrost. Discharge Symp., Sep. 2000, pp. 85-96.

[4] F. Lafon, F. De-Daran, and J. Dupois, "near field immunity cartography method to characterize IC to fields radiated by an ESD," ICONIC, UPC, Barcelona, Spain, Jun. 8-10, 2005.

[5] G.Muchaidze, J.Koo, Q.Cai; T.Li, L, Han, A.Martwick; K.Wang J.Min; J.Drewniak; D.Pommerenke, "Susceptibility Scanning as a Failure Analysis Tool for System-
Level Electrostatic Discharge (ESD) Problems," IEEE Trans EMC, Vol. .50, No.2, May 2008, pp. 268 - 276

[6] Y. Vives, C. Arcambal, A. Louis, F. Daran, P. Eudeline, B. Mazari, "Modeling magnetic radiations of electronic circuits using near-field scanning method," IEEE Trans. Electron. Vol. 49, No. 2, May 2007, pp. 391-400.

[7] Shaowei Deng, Todd H. Hubing, and Daryl G. Beetner, "Using TEM cell measurements to estimate the maximum radiation from PCBs with cables due to magnetic field coupling," IEEE Trans. Electron., Vol.. 50, No. 2, May 2008.

[8] H. Onomae, H. Ito, T. Ishida, T. Eguchi, Y. Nagasawa, "Electromagnetic field characteristics of injection probes," IEEE EMC Int. Symp, May 1999, pp. 55-58.

[9] W.Pan, D.Pommerenke, S.Xu, J.Jia, "PCB ground fill design guidelines for radiated EMI," IEEE EMC Symp. Detroit 2008, in press.

[10] Tun Li, Yong Cheh Ho, David Pommerenke, "Orthogonal loops probe design and characterization for near-field measurement," IEEE EMC Symp. Detroit 2008, in press. 